\title{
BLACKBIRD BANDING RESULTS
}

\author{
by J. A. SLIMMON*
}

In 1963, under the direction of Dr. B. Gollop, Canadian Wildlife Serice, I began banding Red-winged Blackbirds and Yellow-headed Blackirds on marshes near Saskatoon. Only estlings and local young which had ust recently left the nest were banded vith emphasis placed on finding Redvinged nestlings rather than Yelloweaded nestlings. In spite of this aproach however, in two of the subequent 11 years, more Yellow-headed estlings were banded than Redvinged.

Table 1 shows the number banded in ach of the 11 years since 1963, the umber of birds subsequently ecovered, and the year of each ecovery. All recoveries are of Redinged Blackbirds.

Table 2 provides the detail of each ecovery. The exact recovery date of ird \#5 is uncertain. The only date vailable to the Patuxent Wildlife enter was the postmark on the letter which the band was returned.

A review of these recoveries show lat three are within year recoveries. ecovery numbers 4 and 6 were found ead at the same slough on which they ere banded, while number 3 was ecaptured at a banding slough.

Recovery pattern of numbers 1,2 id 5 is interesting. The first two uumber 1 and 2) suggest that Redinged Blackbirds banded in the askatoon area return to the same

2526 Hanover Avenue

jaskatoon, Saskatchewan. 'general' area. If this is correct, number 1 and 5 returned four times while number 2 returned three times. Recovery number 5 indicates that some Saskatoon Red-winged Blackbirds migrate as far south as Mexico. The general recovery area of number 5 was near Carranza some 1,180 miles southeast of the California-Mexico border and 560 miles southwest of the Texas-Mexico border.

A conclusion which could be reached from Table 1 is that in a period of 12 years (in ten of which birds were banded) of the 1,467 nestling Red-winged and Yellowheaded Blackbirds banded only a $0.20 \%$ of the birds banded were recovered. This suggests that, if one were to hope for one recovery per year, an average of 500 nestling Blackbirds would have to be banded each year.

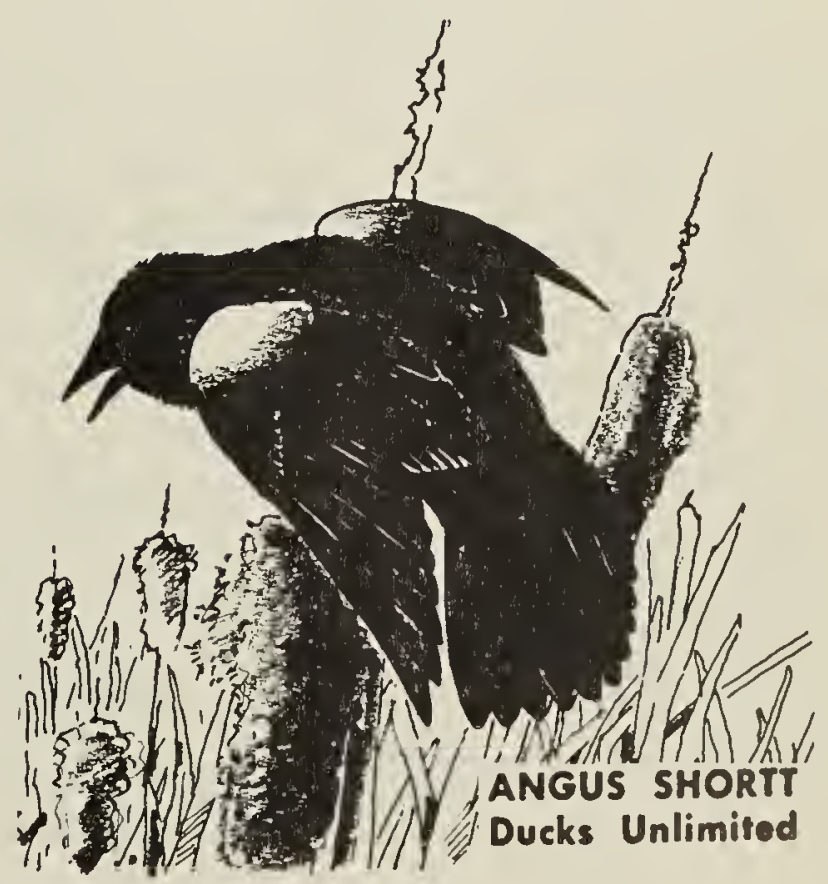


Table 1.

Banding and Recoveries of Blackbirds

$\begin{array}{lrrrrr}\text { Year } & \text { Yellow-headed } & \text { Red-winged } & \text { Total } & \text { Recoveries } & \begin{array}{r}\text { Year of } \\ \text { recovery }\end{array} \\ 1963 & 123 & - & 123 & 1 & 1967 \\ 1964 & 16 & 24 & 40 & - & - \\ 1965 & 62 & 145 & 207 & - & - \\ 1966 & 52 & 42 & 94 & - & - \\ 1967 & 106 & 26 & 132 & - & - \\ 1968 & - & - & -125 & - & - \\ 1969 & 93 & - & 71 & - & - \\ 1970 & 71 & - & 249 & - & 1974 \\ 1971 & - & 116 & 250 & 1 & 1974 \\ 1972 & 157 & 75 & 176 & - & 6 \\ 1973 & 134 & 101 & 552 & 1,467 & \end{array}$

Table 2.

Details on recoveries of banded Blackbirds from Saskatoon

\begin{tabular}{|c|c|c|c|c|c|c|c|}
\hline $\begin{array}{c}\text { Re- } \\
\text { covery } \\
\text { No. }\end{array}$ & $\begin{array}{c}\text { Date } \\
\text { banded }\end{array}$ & $\begin{array}{c}\text { Location } \\
\text { of } \\
\text { banding }\end{array}$ & $\begin{array}{l}\text { Date } \\
\text { recov- } \\
\text { ered }\end{array}$ & $\begin{array}{l}\text { Location } \\
\text { of } \\
\text { recovery }\end{array}$ & $\begin{array}{c}\text { Recovered } \\
\text { by }\end{array}$ & $\begin{array}{l}\text { Age at } \\
\text { recovery }\end{array}$ & $\begin{array}{c}\text { State } \\
\text { at } \\
\text { recovery }\end{array}$ \\
\hline 1 & $22 / 6 / 63$ & $\begin{array}{l}\text { Hudson } \\
\text { Bay } \\
\text { Marsh }\end{array}$ & $8 / 9 / 67$ & $\begin{array}{l}4 \mathrm{mi} . \mathrm{N} . \\
\text { of Leroy, } \\
\text { Sask. }\end{array}$ & $\begin{array}{l}\text { Cal } \\
\text { Torwalt, } \\
\text { Leroy, } \\
\text { Sask. }\end{array}$ & $\begin{array}{l}4 \text { yrs., } \\
2-1 / 2 \\
\text { mos. }\end{array}$ & shot \\
\hline 2 & $21 / 6 / 65$ & $\begin{array}{l}\text { Hudson } \\
\text { Bay } \\
\text { Marsh }\end{array}$ & $19 / 5 / 68$ & $\begin{array}{l}2 \mathrm{mi} . \text { E. } \\
\text { of Warman, } \\
\text { Sask. }\end{array}$ & $\begin{array}{l}\text { Rick } \\
\text { Moore, } \\
\text { Saskatoon, } \\
\text { Sask. }\end{array}$ & $\begin{array}{l}2 \text { yrs., } \\
11 \text { mos. }\end{array}$ & shot \\
\hline 3 & $19 / 6 / 73$ & $\begin{array}{l}\text { Gevelot } \\
\text { Slough }\end{array}$ & $7 / 7 / 73$ & $\begin{array}{l}\text { Same } \\
\text { location }\end{array}$ & $\begin{array}{l}\text { Stuart } \\
\text { Rosmussen, } \\
\text { Saskatoon, } \\
\text { Sask. }\end{array}$ & 18 days + & $\begin{array}{l}\text { caught } \\
\text { and } \\
\text { released }\end{array}$ \\
\hline 4 & $2 / 7 / 73$ & $\begin{array}{l}\text { Clarence } \\
\text { Avenue, } \\
\text { South }\end{array}$ & $31 / 7 / 73$ & $\begin{array}{l}\text { Same } \\
\text { location. }\end{array}$ & $\begin{array}{l}\text { Vernon } \\
\text { Linn, } \\
\text { Saskatoon, } \\
\text { Sask. }\end{array}$ & 29 days + & $\begin{array}{l}\text { found } \\
\text { dead }\end{array}$ \\
\hline 5 & $30 / 6 / 69$ & $\begin{array}{l}\text { North } \\
\text { Airport } \\
\text { Slough }\end{array}$ & $? / 4 / 74$ & $\begin{array}{l}\text { Carranza, } \\
\text { Mexico. }\end{array}$ & $\begin{array}{l}\text { Ramiro } \\
\text { Rodriguez, } \\
\text { Mexico. }\end{array}$ & $\begin{array}{l}4 \text { yrs., } \\
9 \text { mos. }\end{array}$ & shot \\
\hline 6 & $23 / 6 / 74$ & $\begin{array}{l}\text { Hudson } \\
\text { Bay } \\
\text { Marsh }\end{array}$ & $27 / 6 / 74$ & $\begin{array}{l}\text { Same } \\
\text { location. }\end{array}$ & $\begin{array}{l}\text { Dean } \\
\text { Bisset, } \\
\text { Saskatoon, } \\
\text { Sask. }\end{array}$ & 4 dayst & $\begin{array}{l}\text { found } \\
\text { dead }\end{array}$ \\
\hline
\end{tabular}

